\title{
Optimal Bandwidth Allocation of Multiple VBR Video Sources with Priorities ${ }^{1}$
}

\author{
Hon-Wai Chu \\ Department of Electrical and Electronic Engineering \\ The Hong Kong University of Science \& Technology \\ Clear Water Bay, Hong Kong \\ Danny H.K. Tsang \\ Department of Electrical and Electronic Engineering \\ The Hong Kong University of Science \& Technology \\ Clear Water Bay, Hong Kong

\begin{abstract}
Liming Liu
Department of Industrial Engineering

The Hong Kong University of Science \& Technology

Clear Water Bay, Hong Kong
\end{abstract}

March 1994

1. This research was supported in part by HKUST's UPGC Research Infrastructure Grant (RI92/93.EG02) and in part by a Hong Kong Telecom Institute of Information Technology grant (HKTIIT93/94.EG01). 


\begin{abstract}
Using variable bit rate (VBR) coding allows ATM networks to transmit video efficiently by statistical multiplexing. The two-layer coding schemes which separate the coded bits into two priority streams are particularly well-suited for ATM networks. Separation is achieved by giving different access priorities of the shared buffer to the two streams. In this paper, we derive formulae to determine the cell loss rates of the two priority streams under three different access control policies. Based on the results obtained, we then study the trade-off of the cell loss rates as the parameters of the control policies vary. We also consider the problem of optimal bandwidth allocation in which the minimum required capacity, the buffer size, and the parameter of the access control are determined while the quality of service such as cell loss rates and maximum cell delay of multiple VBR video are satisfied.
\end{abstract}




\section{Introduction}

Broadband integrated services digital networks (B-ISDN) allow a flexible integration of a wide variety of services with very different traffic characteristics. Among these services, video is expected to be a major traffic component which demands relatively large network bandwidth and stringent delay requirements. The need to support HDTV or real-time video in a multimedia environment places a heavy burden on B-ISDN.

Asynchronous transfer mode (ATM) based B-ISDN have recently attracted much research attention. ATM, a fast packet-switching technique, allocates bandwidth only when needed. It can achieve higher channel efficiency by statistically multiplexing a number of calls to exploit the burstiness of the sources. The basic transport unit in ATM networks is a 53-byte cell that consists of 5-byte control and 48-byte information data. A major issue in transmitting video over ATM networks is to guarantee users's quality of service, such as cell loss rate and delay jitter, with high bandwidth efficiency. Cells may be lost because of transmission error or buffer overflow and the tolerance of cell loss greatly depends on the type of call.

Traditionally most of the digital coding schemes [11] for video have assumed a channel with fixed capacity. By using a feedback mechanism, the video codec reduces the amount of bits generated to avoid buffer overflow when the output buffer reaches a certain threshold value. The reduction of bit rate ensures that the total generated bit rate is below the fixed channel capacity, however, it results in the fluctuation of video quality. With the future availability of ATM networks, it is widely believed that constant quality of video can be maintained by the statistical multiplexing nature of ATM networks and appropriate network controls.

Variable bit rate (VBR) techniques [7,8] are well suited for coding video sources because they can take advantage of the statistical multiplexing nature of ATM networks while maintaining a uniform picture quality. Of the different VBR coding schemes proposed, the two-layer coding scheme $[2,10]$ works nicely with the ATM standard which specifies the support of two levels of priority via the use of a cell loss priority (CLP) bit in the header of each ATM cell. The CLP bit distinguishes the two different priorities of traffic by letting the high priority stream have full access to the shared buffer while providing only limited access to the low priority stream. The two-layer coding separates the coded bits into two streams: the basic stream (high priority) and the enhancement stream (low priority). During network congestion, the enhancement stream would suffer some cell loss with acceptable degradation of video quality while the basic stream 
should experience no cell loss or very minimal cell loss. This is achieved by giving different access priorities of the shared buffers to the two priority streams $[3,5]$.

In this paper, we investigate the problem of resource allocation for a multiplexer supporting VBR video sources with priorities. We determine the optimal capacity and buffer size required to guarantee the quality of service (QoS) requirements of the videos. Particularly, the video sources are heterogeneous with different input characteristics and different QoS requirements. In order to simplify the analysis, we introduce the Markov Modulated Deterministic Process (MMDP) to model a VBR video source. MMDP basically assume that the cell arrival rates of the source are fixed within each frame but the rates can change from frame to frame according to an underlying Markov Chain. Consequently, we can analyze the queueing system with input streams of two different priorities.

The organization of the paper is as follows: we first introduce MMDP and then present an analysis of the resulting queueing system. An efficient algorithm to compute the cell loss probability will be given. We then derive the cell loss rates of the two traffic streams under three different access control policies. In Section 3, we show our numerical results for the three different schemes. Based on the results obtained, we then study the trade-off of the cell loss rates as the parameters of the control policies vary. In Section 4, we consider the problem of determining the optimal capacity and buffer required to guarantee the QoS requirements of the video sources. Moreover, an iterative algorithm is proposed to find the optimal resource allocation. Finally, we summarize the results in the conclusion.

\section{VBR videos with priorities}

\subsection{MMDP Model}

We first assume that the video is limited to $M$ allowable constant arrival rates. We then model the rate process of each video source as a discrete-time Markov chain $\left\{X_{n}\right\}$ with state space $\Omega=$ $\{1,2, \ldots, M\}$ and a $M \times M$ transition probability matrix. The constant transition time between states corresponds directly to the deterministic time between two successive frames. The period of a frame is assumed to be $T$ seconds and the frame rate is thus $1 / T$. The transition probabilities can be calculated either from the statistical parameters of video sequences or by direct measurement [8, 9]. The value of $M$ is a design parameter which determines the fineness of the quantization levels. 
It is found in $[8,9]$ that eight bins or levels are usually sufficient for the representation of video and hence we choose a Markov chain with eight states, (i.e., $M=8$ ), in this paper.

A similar model for the video arrival process is also proposed in [4] in which $M=8$ and the transition probability matrix is obtained by direct measurement. The Chernoff bound was proposed in [4] to do bandwidth allocation for homogeneous sources without priority in a bufferless system. However, it is well known [1] that the cell loss probability can be expressed as the sum of two terms, which correspond to the cell-level and burst-level congestion regions. For small buffer ( $<10$ cells in size), the cell level congestion dominates and causes significant cell loss. As a result, the bufferless analysis gives a conservative estimate for practical systems with buffer. In this paper, we analyze a more practical case, namely, heterogeneous sources with priority in a buffered system.

Suppose there are $S$ independent video sources with transition probability matrix $\mathbf{P}^{i}$ for $i=$ $1, \ldots, N$. While in state $j \in \Omega$, the $i$ th video source generates deterministic cell arrivals at the rates of $B_{i, j}{ }^{(1)}$ and $B_{i, j}{ }^{(2)}$ for the high and low priority streams, respectively. These streams are multiplexed and put into a common buffer of size $K$ to await transmission. The aggregate stream can be modeled by a Markov Chain with state space $\Lambda \equiv\left\{x=\left(x_{1}, x_{2}, \ldots, x_{\mathrm{S}}\right) \mid 1 \leq x_{\mathrm{i}} \leq \mathrm{M}, \mathrm{i}=1,2, \ldots, \mathrm{S}\right\}$. If the aggregate source is in state $x$, the corresponding aggregate arrival rates for the two priority streams are respectively:

$$
B_{x}^{(1)}=\sum_{i=1}^{S} B_{i, x_{i}}^{(1)} \text { and } B_{x}^{(2)}=\sum_{i=1}^{S} B_{i, x_{i}}^{(2)}
$$

The transition probability $p_{x, y}$, where $x, y \in \Lambda$, of the aggregate arrival process is given by:

$$
p_{x, y}=\prod_{i=1}^{S} p_{x_{i}, y_{i}}
$$

where $\mathbf{P} \equiv\left[p_{x, y}\right]$ and $\mathbf{P}^{i} \equiv\left[p_{x_{i}, y_{i}}\right]$

To simplify the notation, denote the vectors $x(x \in \Lambda)$ in the usual lexicographical order as follows:

$$
\begin{gathered}
x=(1,1, \ldots, 1) \equiv w_{1} \\
x=(1,1, \ldots, 2) \equiv w_{2} \\
\ldots \\
x=(M, M, \ldots, M) \equiv w_{\lambda}
\end{gathered}
$$


where $\lambda=M^{S}$ and thus the state space $\Lambda=\left\{\mathrm{w}_{1}, \mathrm{w}_{2}, \ldots, \mathrm{w}_{\lambda}\right\}$.

The aggregate arrival rate $B_{x}$ is equal to the sum of $B_{x}^{(1)}$ and $B_{x}^{(2)}$. The capacity of the channel is assumed to be $C$ cells per second. A threshold $K_{1}$ less than $K$ is established to control the admission of the low priority stream to the buffer. Here we are interested in determining the cell loss rates of the two priority streams under three different information-based access control schemes (see Table 1). The controlled input rate $R$ is defined as the allowed admission rate of the video streams into the buffer. We assume that the buffer content can be monitored in all three schemes and the decision to accept or reject the low priority cells is made only at the beginning of a frame time. They differ in the amount of information available to the system and hence execute different cell admission rules. In Scheme I, no knowledge of the input rates of the two priority streams are available and the low priority cells are rejected when the buffer content exceeds $K_{1}$. While in Scheme II, the total input rate $B_{x}$ is known and the low priority cells are rejected only when the buffer content exceeds $K_{1}$ and $B_{x}$ is larger than the capacity $C$. In Scheme III, the individual rates of the two priority streams are also known and the low priority cells are admitted according to the rule specified in Table 1. On the other hand, all the arriving cells are accepted into the buffer when the buffer content is less than $K_{1}$. The high priority cells are lost only when the buffer is full.

The aggregate arrival process of the video source shall be referred to as a Markov Modulated Deterministic Process (MMDP), which is completely defined by the matrix $\mathbf{P}$, the frame rate $1 / T$ and the rates $\mathrm{B}_{x}^{(\mathrm{j})}, x \in \Lambda, \mathrm{j}=1,2$.

\begin{tabular}{|c|c|c|c|}
\hline $\begin{array}{l}\text { Buffer } \\
\text { content }\end{array}$ & $\begin{array}{l}\text { Scheme I } \\
\text { No knowledge of } \\
\text { input rates }\end{array}$ & $\begin{array}{l}\text { Scheme II } \\
\text { Only total input rate } \\
B_{x}=B_{x}^{(1)}+B_{x}^{(2)} \text { is } \\
\text { known }\end{array}$ & $\begin{array}{c}\text { Scheme III } \\
\text { Individual rates } B_{x}^{(1)} \text { and } B_{x}^{(2)} \\
\text { are known }\end{array}$ \\
\hline$>K$ & $R=B_{x}^{(1)}$ & $R= \begin{cases}B_{x}^{(1)} & \text { if } \quad B_{x}>C \\
B_{x} & \text { if } \quad B_{x} \leq C\end{cases}$ & $R=\left\{\begin{array}{ccc}B_{x}^{(1)} & \text { if } & B_{x}>C, B_{x}^{(1)}>C \\
C & \text { if } & B_{x}>C, B_{x}^{(1)} \leq C \\
B_{x} & \text { if } & B_{x} \leq C\end{array}\right.$ \\
\hline$\leq K_{1}$ & $R=B_{x}$ & $R=B_{x}$ & $R=B_{x}$ \\
\hline
\end{tabular}

Table 1: Controlled input rate $R$ of the three schemes 


\subsection{The MMDP/D/1/K Queueing System}

We use a two-dimensional Markov chain $\left(X_{n}, Y_{n}\right)$ to model the system where $X_{n}$ is the MMDP arrival process as defined in the previous section and $Y_{n}$ is the buffer content at the beginning of the $n^{\text {th }}$ frame. Let $q_{x k, y h}$ be the transition probability from state $(x, k)$ to state $(\mathrm{y}, h)$ of the Markov chain. Then,

$$
q_{x k, y h}=P\left(Y_{n+1}=h \mid X_{n}=x, Y_{n}=k\right) p_{x, y}=a_{k h}^{x} p_{x, y}
$$

where $a_{k h}^{x} \equiv P\left(Y_{n+1}=h \mid X_{n}=x, Y_{n}=k\right)$ and $p_{x, y}$ is an element of the transition matrix $\mathbf{P}$ of the video sources. Let $\mathbf{A}^{x} \equiv\left[a_{k h}^{x}\right]$ and $\mathbf{Q} \equiv\left[q_{x k, y h}\right]$. In the usual lexicographical order $\left\{\left(\mathrm{w}_{1}, 0\right), \ldots,\left(\mathrm{w}_{1}, K\right), \ldots,\left(\mathrm{w}_{\lambda}, 0\right), \ldots\left(\mathrm{w}_{\lambda}, K\right)\right\}$, we can partition $\mathbf{Q}$ into blocks, each of which is a $(K+1) \times(K+1)$ probability matrix:

$$
Q=\left[\begin{array}{cccc}
A^{w_{1}} p_{w_{1} w_{1}} & A^{w_{1}} p_{w_{1} w_{2}} & \ldots & A^{w_{1}} p_{w_{1} w_{\lambda}} \\
A^{w_{2}} p_{w_{2} w_{1}} & A^{w_{2}} p_{w_{2} w_{2}} & \ldots & A^{w_{2}} p_{w_{2} w_{\lambda}} \\
\ldots & \ldots & \ldots & \ldots \\
A^{w_{\lambda}} p_{w_{\lambda} w_{1}} & A^{w_{\lambda}} p_{w_{\lambda} w_{2}} & \ldots & A^{w_{\lambda}} p_{w_{\lambda} w_{\lambda}}
\end{array}\right]
$$

The structure of $\mathbf{A}^{x}$ depends on the control schemes that are used in the system. As an example, we determine matrix $\mathbf{A}^{x}$ for Scheme I. The $\mathbf{A}^{x}$ matrix for Schemes II and III can be determined similarly and are summarized in Appendix A. In Scheme I, the system has no knowledge of the arrival rates and can only monitor the buffer content to determine the controlled input rate. If buffer content exceeds the threshold $K_{1}$, all the low priority cells are rejected and only high priority cells are admitted. Otherwise, all the cells are accepted. This control scheme is the same as the one discussed in [3]. To compute $a_{k h}^{x}$, we consider three cases.

Case (1), $B_{x} \leq C$ :

$$
a_{k h}^{x}= \begin{cases}1 & k \leq K_{1}, h=\max \left(0, k-\left\lfloor\left(C-B_{x}\right) T\right\rfloor\right) \\ 1 & k>K_{1} \text { and } T_{1}>T, \quad h=k-\left\lfloor\left(C-B_{x}^{(1)}\right) T\right\rfloor \\ 1 & k>K_{1} \text { and } T_{1} \leq T, h=\max \left(0, K_{1}-\left\lfloor\left(C-B_{x}^{(1)}\right)\left(T-T_{1}\right)\right\rfloor\right) \\ 0 & \text { otherwise }\end{cases}
$$


where $T_{1}=\left(k-K_{1}\right) /\left(C-B_{x}^{(1)}\right)$.

Case (2), $B_{x}>C$ and $B_{x}^{(1)}<C$ :

$$
a_{k h}^{x}= \begin{cases}1 & k \leq K_{1}, h=\min \left(K_{1}, k+\left\lfloor\left(B_{x}-C\right) T\right\rfloor\right) \\ 1 & k>K_{1}, h=\max \left(K_{1}, k-\left\lfloor\left(C-B_{x}^{(1)}\right) T\right\rfloor\right) \\ 0 & \text { otherwise }\end{cases}
$$

Case (3), $B_{x}>C$ and $B_{x}^{(1)} \geq C$ :

$$
a_{k h}^{x}= \begin{cases}1 & k \leq K_{1} \text { and } T_{2}>T, h=k+\left\lfloor\left(B_{x}-C\right) T\right\rfloor \\ 1 & k \leq K_{1} \text { and } T_{2} \leq T, h=\min \left(K, K_{1}+\left\lfloor\left(B_{x}^{(1)}-C\right)\left(T-T_{2}\right)\right\rfloor\right) \\ 1 & k>K_{1}, \quad h=\min \left(K, k+\left\lfloor\left(B_{x}^{(1)}-C\right) T\right\rfloor\right) \\ 0 & \text { otherwise }\end{cases}
$$

where $T_{2}=\left(K_{1}-k\right) /\left(B_{x}-C\right)$.

Note that each row of $\mathbf{A}^{x}$ contains exactly one non-zero element which has a value of 1 . Thus, the matrix $\mathbf{A}^{x}$ is very sparse.

We define the limiting probability $\pi_{x k} \equiv \lim _{n \rightarrow \infty} P\left(X_{n}=x, Y_{n}=k\right)$ and the vector $\pi_{x} \equiv\left(\pi_{x 0}\right.$, $\left.\pi_{x l}, \ldots, \pi_{x K}\right)$. It can be shown that $\left\{\left(X_{n}, Y_{n}\right), n \geq 0\right\}$ is ergodic and therefore, $\pi_{x}, x \in \Lambda$, are uniquely determined from the balance equations:

$$
\pi_{x}=\sum_{y \in \Lambda} \pi_{y} A^{y} p_{y, x}, \quad x \in \Lambda
$$

and

$$
\sum_{x \in \Lambda} \pi_{x} e^{T}=1
$$

where $\mathbf{e}$ is a $(K+1)$-dimensional vector of all 1 's and $\mathbf{e}^{\mathrm{T}}$ is its transpose.

The average cell loss probabilities, $\bar{\beta}^{(j)}, \mathrm{j}=1,2$, of the two priority streams can be computed from $\pi_{x}, x \in \Lambda$, by 


$$
\begin{aligned}
\bar{\beta}^{(j)} & =\frac{\text { mean cell loss of stream }(\mathrm{j}) \text { in a frame period }}{\text { mean cell arrivals of stream (j) in a frame period }} \\
& =\left(\sum_{x \in \Lambda k=0} \sum_{x k}^{K} L_{x k}^{(j)} \pi_{x k}\right) /\left(\sum_{x \in \Lambda k=0} \sum_{x k}^{K} N_{x k}^{(j)} \pi_{x k}\right), \quad j=1,2,
\end{aligned}
$$

where $L_{x k}^{(j)}$ and $N_{x k}^{(j)}$ are the number of cell lost and the number of cell arrivals, respectively, of priority stream $j$ given that the system is in state $(x, k)$ at the beginning of a frame.

The number of cell arrivals $N_{x k}^{(j)}$ for the stream $j$ in a frame period is fixed and is equal to $\left\lfloor B_{x}^{(j)} T\right\rfloor, j=1,2$.

We now consider the computation of $L_{x k}^{(j)}$ for the two priority streams for Scheme I. The value of $L_{x k}^{(j)}$ for Schemes II and III traffic can be derived in a similar way.

For the high priority stream, cells are lost only when $B_{x}^{(1)}>C$ and the buffer is full. Thus

$$
L_{x k}^{(1)}= \begin{cases}\max \left(0,\left\lfloor\left(B_{x}^{(1)}-C\right) T\right\rfloor-K+k\right) & k>K_{1} \\ \max \left(0,\left\lfloor\left(B_{x}^{(1)}-C\right) \cdot\left(T-T_{2}\right)\right\rfloor-K+K_{1}\right) & k \leq K_{1} .\end{cases}
$$

For the low priority stream, we have three different cases:

i) $B_{x} \leq C$,

$$
L_{x k}^{(2)}= \begin{cases}\left\lfloor B_{x}^{(2)} \cdot \min \left(T, T_{1}\right)\right\rfloor & k>K_{1} \\ 0 & k \leq K_{1},\end{cases}
$$

ii) $B_{x}>C$ and $B_{x}^{(1)}<C$,

$$
L_{x k}^{(2)}= \begin{cases}\left\lfloor B_{x}^{(2)} T_{1}\right\rfloor+\left\lfloor\left(B_{x}-C\right) \cdot\left(T-T_{1}\right)\right\rfloor & k>K_{1}, T>T_{1} \\ \left\lfloor B_{x}^{(2)} T\right\rfloor & k>K_{1}, T \leq T_{1} \\ \left\lfloor\left(B_{x}-C\right) \cdot \max \left(0, T-T_{2}\right)\right\rfloor & k \leq K_{1},\end{cases}
$$


iii) $B_{x}>C$ and $B_{x}^{(1)} \geq C$,

$$
L_{x k}^{(2)}= \begin{cases}\left\lfloor B_{x}^{(2)} T\right\rfloor & k>K_{1} \\ \left\lfloor B_{x}^{(2)} \cdot \max \left(0, T-T_{2}\right)\right\rfloor & k \leq K_{1} .\end{cases}
$$

For practical applications, we are not only interested in the average cell loss probabilities but also the individual cell loss probabilities for each video source. During each frame period, cell arrivals, cell departures, and cell loss are all deterministic. For each of the two priority streams, the total cell arrivals from the $i$ th video in a frame period is simply equal to $\left\lfloor B_{i, x_{i}}^{(j)} T\right\rfloor$. Similarly, the total cell loss from the $i$ th video is a fraction of the total cell loss in the same period, which is equal to $B_{i, x_{i}}^{(\mathrm{j})} / B_{x}{ }^{(\mathrm{j})}$. Hence the individual cell loss probability $\beta_{i}^{(j)}$ for the $i$ th video is given by

$$
\beta_{i}^{(j)}=\left(\sum_{x \in \Lambda} \sum_{k=0}^{K} L_{x k}^{(j)} \pi_{x k} \frac{B_{i, x_{i}}^{(j)}}{B_{x}^{(j)}}\right) /\left(\sum_{x \in \Lambda k=0} \sum_{k=0}^{K}\left\lfloor B_{i, x_{i}}^{(j)} T\right\rfloor \pi_{x k}\right), \quad j=1,2 \text { and } i=1, \ldots, M
$$

\subsection{Iterative Algorithm}

The limiting probabilities $\pi_{x}, x \in \Lambda$, can be obtained using standard numerical methods by solving the system of linear equations (4) and (5). However, substantial savings in computation can be obtained by exploiting the special properties of the Markov chain $\left\{\left(X_{n}, Y_{n}\right), n \geq 0\right\}$. In the following we discuss two special properties of the chain and develop an efficient algorithm to compute the cell loss probabilities by making use of these properties.

First, since $\mathbf{A}^{x}$ are stochastic matrices (i.e., $\mathbf{A}^{x} \mathbf{e}^{\mathrm{T}}=\mathbf{e}^{\mathrm{T}}$ ), we observe from the transition matrix $\mathbf{Q}$ that the Markov chain $\left\{\left(X_{n}, Y_{n}\right), n \geq 0\right\}$ is lumpable [4] with respect to the partition $\left(\Theta_{\mathrm{w}_{1}}, \ldots\right.$, $\left.\Theta_{w_{\lambda}}\right)$, where $\Theta_{w_{i}} \equiv\left\{\left(w_{i}, 0\right),\left(w_{i}, 1\right), \ldots,\left(w_{i}, K\right), i=1, \ldots, \lambda\right\}$. Based on the theory given in [4], we can show that the lumped chain has probability transition matrix $\mathbf{P}$. Its limiting probabilities $\sigma_{x}=\pi_{x} \mathbf{e}^{\mathrm{T}}$ are given by

where $\sigma_{i, x_{i}}$ are determined from

$$
\sigma_{x}=\prod_{i=1}^{S} \sigma_{i, x_{i}}
$$

$$
\begin{aligned}
& \sigma_{i}=\sigma_{i} \mathbf{P}^{i} \\
& \text { and } \quad \sum_{j=1}^{M} \sigma_{i, j}=1, \quad i=1, \ldots, S
\end{aligned}
$$


and we define $\sigma_{i} \equiv\left(\sigma_{i, 1} \sigma_{i, 2}, \ldots, \sigma_{i, M}\right)$.

The system of equations in (9) can be solved by standard numerical methods once the matrix $\mathbf{P}^{i}$ is known. The fact that the lumped probability $\pi_{x} \mathbf{e}^{\mathrm{T}}$ can be computed without the knowledge of $\pi_{x}$ is the main factor that speeds up the iterative algorithm to be discussed below.

Second, each row of $\mathbf{A}^{x}$ contains exactly one non-zero element which has a value of 1 . Hence, the computation of the term $\pi_{x} \mathbf{A}^{x}$ is greatly reduced by using sparse matrix computation.

To make use of the above two special properties, we develop an iterative algorithm based on the Gauss-Seidel method. However, immediately after updating $\pi_{i}$, we perform a local normalization by scaling the elements in $\pi_{x}$ so that $\pi_{x} \mathrm{e}^{\mathrm{T}}=\sigma_{x}$, where $\sigma_{x}$ is pre-calculated using Equations (8) \& (9). Therefore, throughout the computation, the aggregate probabilities $\pi_{x} \mathrm{e}^{\mathrm{T}}$ are always accurate.

This iterative procedure is summarized in the algorithm shown in the box. In the algorithm, $\pi_{x}(0)$ is uniformly initialized and $\varepsilon$ is a pre-specified error tolerance.

\section{Algorithm for Determining Individual Cell Loss Probabilities}

1. $\mathrm{k} \leftarrow 0 ; \bar{\beta}^{(j)}(0) \leftarrow 0, \mathrm{j}=1,2$; initialize $\pi_{\mathrm{x}}(0), \mathrm{x} \in \Lambda$; compute $\sigma_{x}$ using (8) \& (9); and compute $\mathbf{A}^{x}$ using (1), (2) \& (3).

2. For $\mathrm{x} \in \Lambda$, compute

$$
\begin{aligned}
& \pi_{x}(k+1) \leftarrow \sum_{y<x} \pi_{y}(k+1) A^{y} p_{y, x}+\sum_{y \geq x} \pi_{y}(k) A^{y} p_{y, x} \\
& \pi_{x}(k+1) \leftarrow \frac{\sigma_{x} \pi_{x}(k+1)}{\pi_{x}(k+1) \mathrm{e}^{\mathrm{T}}} \quad \text { (local normalization). }
\end{aligned}
$$

3. Compute the average cell loss probability $\bar{\beta}^{(j)}(k+1), j=1,2$, using (6).

4. If $\max _{j=1,2}\left(\frac{\left|\bar{\beta}^{(j)}(k+1)-\bar{\beta}^{(j)}(k)\right|}{\bar{\beta}^{(j)}(k+1)}\right) \geq \varepsilon \quad$, then $\mathrm{k} \leftarrow \mathrm{k}+1$ and goto step 2 .

5. Compute the individual cell loss prob. $\beta_{i}^{(j)}, \mathrm{j}=1,2, \mathrm{i}=1,2, \ldots, \mathrm{S}$ using (7).

6. Terminate. 
Remark: In general, the above algorithm that employs local normalization will converge to the desired solution within 20 iterations.

\section{Numerical results}

In this section, we examine the performance trade-off between the high and the low priority streams under three different access schemes using the algorithm developed in the previous section. The threshold $K_{1}$ also controls the trade-off in cell loss probability between the high and the low priority streams, whereas the buffer size $K$ determines the trade-off between the cell loss probability of the high priority streams and the maximum cell delay. Since we are only interested in comparing the performance of the three access schemes, which are independent of the number of video sources, we thus consider only the case of a single video source (i.e., $\mathrm{S}=1$ ) to save computational time. We assume that $\mathrm{M}=8$ and the frame rate is 24 frames/s. The cell length is assumed to be 53 bytes and thus the rates have to be divided by 424 to convert them back into cells/second. The transition matrix $\mathbf{P}^{(1)}$ of the video source is given in Appendix B.

Numerical results are summarized in Figs. $1 \& 2$. We note that Schemes I \& II have almost identical performance, while Schemes I \& III show a larger difference between them which is particularly significant for small values of $K_{1}$. The additional admission of the low priority cells in Scheme III has degraded the service quality of the high priority cells. When $K_{1}$ is closed to $K$, the differences between the three schemes diminish. Figure 2 shows that the cell loss probability of the low priority stream increases only slightly with $K$, when $K_{1}$ is fixed. This agrees with the observation cited in [3]. On the other hand, the increase in $K$ continues to reduce the cell loss probability of the high priority stream. In addition, the cell loss probabilities of both priority streams decrease with increasing $C$. This monotonicity property allows the minimum $C$ to be searched for by the bisection method when $K$ and $K_{1}$ are fixed.

After extensive simulation, we have also found that the limiting distributions of buffer contents in Schemes I \& II show a tri-modal behavior. That is, the buffer is highly likely to be either empty, at $K_{1}$ or full. Nevertheless, the limiting distribution of buffer content in Scheme III is spreading out in the region $K_{1}$ to $K$ and does not show the tri-modal behavior.

Remark: We have also compared the results with three video sources (i.e., $\mathrm{S}=3$ ) and found similar properties as in the single video case. 


\section{Optimal design of priority systems}

Suppose that there are $\mathrm{S}$ video sources and the $i$ th source has a maximum cell loss probability requirement of $\left(\alpha_{i}^{(1}, \alpha_{i}^{(2)}\right)$ for the two priority streams and a maximum cell delay requirement of $D_{\mathrm{i}}$. Here we are interested in determining the minimum capacity $C$ and the corresponding system parameters $K$ and $K_{1}$ to satisfy all the individual QoS requirements. This can also be considered as determining the equivalent bandwidth in the context of call admission control.

We define $\alpha^{(1)} \equiv\left(\alpha_{1}^{(1)}, \alpha_{2}^{(1)}, \ldots, \alpha_{S}^{(1)}\right), \alpha^{(2)} \equiv\left(\alpha_{1}^{(2)}, \alpha_{2}^{(2)}, \ldots, \alpha_{S}^{(2)}\right)$ and $D \equiv \min _{i}\left\{D_{i}\right.$ for $i=$ $1, \ldots, \mathrm{S}\}$. The maximum cell delay can be expressed as $K / C$. As a result, we can formulate the problem in the form of an optimization problem given below:

$$
\begin{gathered}
\min C \quad \text { such that } \\
\beta^{(1)}\left(C, K, K_{1}\right) \leq \alpha^{(1)} \\
\beta^{(2)}\left(C, K, K_{1}\right) \leq \alpha^{(2)} \\
K / C \leq D \\
0 \leq K_{1} \leq K
\end{gathered}
$$

where $\beta^{(j)} \equiv\left(\beta_{1}^{(j)}, \beta_{2}^{(j)}, \ldots, \beta_{S}^{(j)}\right)$ for $\mathrm{j}=1,2$.

Note that we adopt the following conventions for vector inequalities in this paper.

$$
\begin{aligned}
& \beta^{(j)} \leq \alpha^{(j)} \Rightarrow \beta_{i}^{(j)} \leq \alpha_{i}^{(j)}, \quad \text { for all } i, i=1,2, \ldots, S \\
& \beta^{(j)}>\alpha^{(j)} \Rightarrow \beta_{i}^{(j)}>\alpha_{i}^{(j)}, \quad \text { for some } i, \quad i=1,2, \ldots, S
\end{aligned}
$$

Before trying to solve this minimization problem, we would like to investigate the interaction among the three parameters $\left(C, K, K_{1}\right)$. Suppose $C(\mathrm{i})$ is the minimal $C$ resulting from some iterative searching algorithm at the $\mathrm{i}^{\text {th }}$ step. Using $C(\mathrm{i})$, we can set the new $K(\mathrm{i}+1)=D \cdot C(\mathrm{i})$ and the new $K_{1}$ will be limited to the range $0 \leq K_{1} \leq K(\mathrm{i}+1)$. In turn, we can search for the new $C(\mathrm{i}+1)$ among different minimum values of $C$ for different $K_{1}$ values while keeping $K(i+1)$ fixed.

During the iterations of the optimization algorithm, we encounter three different cases for a given set of $\left(C, K, K_{1}\right)$. 
Case (a): $\quad \beta^{(1)} \leq \alpha^{(1)}$ and $\beta^{(2)}<\alpha^{(2)}$

In this case, at least one of the cell loss probabilities of the high priority streams is satisfied by equality while all that of the low priority streams are less than the specified maximum values. In order to find a smaller value of $C$ while still satisfying the cell loss probability constraints, one would try to reduce the value of $K_{1}$ and thus the new search region is $\left[0, K_{1}\right]$. By decreasing $K_{1}$, $\beta^{(1)}$ would decrease at the expense of increasing $\beta^{(2)}$, as shown in Section 3. Any value of $K_{1}$ in the other region $\left(K_{1}, K\right]$ would definitely result in a larger value of $C$ to satisfy the cell loss probability constraints in this case.

Case (b): $\quad \beta^{(1)}<\alpha^{(1)}$ and $\beta^{(2)} \leq \alpha^{(2)}$,

Similar to the arguments in Case (a), suppose the current $\left(C, K, K_{1}\right)$ gives the results of Case (b). To find a smaller possible value of $C$ for which the cell loss probability constraints continue to be satisfied, the value of $K_{1}$ should be increased (i.e., the search region is $\left[K_{1}, K\right]$ ). By increasing $K_{1}, \beta^{(2)}$ would decrease at the expense of increasing $\beta^{(1)}$.

Case (c): $\quad \beta^{(1)} \leq \alpha^{(1)}$ and $\beta^{(2)} \leq \alpha^{(2)}$,

In this case, changing $K_{1}$ in either direction would violate the cell loss probability constraints and thus require an increase of $C$. Therefore, the search for a better $K_{1}$ to minimize $C$ should be terminated in this case.

When $K$ is fixed, denote the minimal $C$ that satisfies the cell loss probability constraints for a given $K_{1}$ by $C^{*}$. We argue that $C^{*}$ as a function of $K_{1}$ is a convex function so that the bisection method can be used to search for the minimum value of $C^{*}$ for $0 \leq K_{1} \leq K$. One can think of doing a brute-force search starting from $K_{1}=0$ and incrementing $K_{1}$ by one at each step. Initially, the cell loss probability constraints are satisfied as in Case (a) and thus incrementing $K_{1}$ by one would likely result in a smaller value of $C^{*}$. The $C^{*}$ would decrease as $K_{1}$ increases to the first value of $K_{1}$ for which the cell loss probability constraints are satisfied as in Case (b). Further increase of $K_{1}$ starting at this value would increase the value of $C^{*}$ as explained in Case (b). Based on these arguments, we therefore conclude that the bisection method is applicable here to search for the minimum $C^{*}$.

In the following, an iterative algorithm is proposed to find the optimal parameters $\left(C, K, K_{1}\right)$. 
Basically, the algorithm employ the bisection method to search for $K_{1}$ that gives the minimum capacity for a given $K$. Furthermore, for a given set of $K$ and $K_{1}$, another bisection method is used to find the minimum capacity to satisfy the cell loss probability constraints.

\section{Iterative algorithm:}

1. $i \leftarrow 1 ; K(1) \leftarrow\lfloor D \cdot C(0)\rfloor$, where $C(0)$ is the total average input rate of all the source.

2. With $K(i)$ fixed, a new $C(i)$ can be found by using the bisection method of steps

(i) - (iv) below:

i) $a \leftarrow 0, b \leftarrow K(i)$ and $j \leftarrow 0$;

ii) $K_{1}(j) \leftarrow a+\lfloor(b-a) / 2\rfloor$; for the given $K(i)$ and $K_{1}(j)$, find the minimum $C^{*}(j)$ that satisfies $\beta^{(k)} \leq \alpha^{(k)}, k=1,2$, using a bisection method and the algorithm in Sec. 2.3.

iii) if $\beta^{(1)} \leq \alpha^{(1)}$ and $\beta^{(2)}<\alpha^{(2)}$, then $b \leftarrow K_{1}(j)$;

$$
\text { if } \beta^{(1)}<\alpha^{(1)} \text { and } \beta^{(2)} \leq \alpha^{(2)} \text {, then } a \leftarrow K_{1}(j) \text {; }
$$$$
\text { if } \beta^{(1)} \leq \alpha^{(1)} \text { and } \beta^{(2)} \leq \alpha^{(2)} \text {, then goto step (3). }
$$

iv) $j \leftarrow j+1$ and repeat (ii) - (iv) until $K_{1}(j)=K_{1}(j-1)$.

3. $C(i) \leftarrow C^{*}(j)$.

4. $K(i+1) \leftarrow\lfloor D \cdot C(i)\rfloor, i \leftarrow i+1$.

5. Repeat steps (2) - (4) until $K(i)=K(i-1)$.

6. Terminate and the optimal $C(i), K(i)$ and $K_{1}(j)$ are found.

For a typical buffer size of 100 cells, the outer bisection search (step 2) will converge to the optimal C(i) in less than 7 iterations, while the inner bisection search (step ii) normally takes less than 15 iterations to reach the minimum $C^{*}(\mathrm{j})$ for the given $K(\mathrm{i})$ and $K_{1}(\mathrm{j})$. A numerical example is shown in Table 3. In this example, we consider two video sources with specifications given in Table 2. The transition probability matrices of the videos, $\mathbf{P}^{1}$ and $\mathbf{P}^{2}$, are given in Appendix B. A cell size of 53 bytes is assumed. 


\begin{tabular}{|c|c|c|c|c|c|}
\hline video $i$ & $B_{i}^{(1)} \mathrm{Mb} / \mathrm{s}$ & $B_{i}^{(2)} \mathrm{Mb} / \mathrm{s}$ & $\alpha^{(1)}$ & $\alpha^{(2)}$ & $D \mathrm{~ms}$ \\
\hline \hline 1 & $(1.0,2.0,3.0,4.0$, & $(0.5,1.0,1.5,2.0$, & $1.0 \times 10^{-7}$ & 0.08 & 3 \\
& $5.0,6.0,7.0,8.0)$ & $2.5,3.0,3.5,4.0)$ & & & \\
\hline 2 & $(1.3,2.5,3.7,4.9$, & $(0.78,1.5,2.22,2.94$, & $1.0 \times 10^{-7}$ & 0.08 & 3 \\
& $6.1,7.3,8.5,9.7)$ & $3.66,4.38,5.1,5.82)$ & & & \\
\hline
\end{tabular}

Table 2: Specification of the video sources

From Table 3, we see that the optimal $\left(C, K, K_{1}\right)$ is obtained after three iterations of $K$. Moreover, the algorithm converges to within $2 \%$ error relative to the optimal $C$ after only one iteration. This has also been observed in other examples after extensive testing. Since $C(0)$ is the minimum capacity to be allocated and thus $C(1) \geq C(0)$, the set $\left(C, K, K_{1}\right)$ at the end of first iteration would certainly satisfy all the constraints. Consequently, we can get a fast and accurate estimate of $C$ by just running one iteration of $K$, which is particularly useful for cases having a large number of video sources.

Nevertheless, the complexity of the algorithm grows exponentially with the number of video sources and this limits the application of the algorithm. Currently, we are looking into various methods which will reduce the complexity. One possible solution is to group video sources with similar properties together so that fewer states are needed to represent the aggregate source.

\begin{tabular}{|c|c|c|c|}
\hline $\begin{array}{c}\text { iteration } \\
i\end{array}$ & $K$ cells & $K_{1}$ cells & $C$ cells/s \\
\hline \hline 1 & 61 & 50 & 41690 \\
\hline 2 & 125 & 75 & 41620 \\
\hline 3 & 124 & 74 & 41620 \\
\hline
\end{tabular}

Table 3: Iteration of the algorithm with two video sources, $\mathrm{C}(0)=20,500$ cells/s.

\section{Conclusion}

In this paper, we have considered a model for the two-layer coding VBR video sources which can be supported by an ATM network. The video sources are characterized by the input rates of 
the two priority traffic streams and the transition matrix, which are readily available by direct measurement. The model is simple enough to be extended to the case of heterogenous video sources. Based on the model, we study the performance of multiple video sources multiplexed into an ATM multiplexer. An algorithm is proposed to compute both the average cell loss probabilities of the resulting queueing system and the individual cell loss probabilities of each video source. This allows us to analyze the problem of multiplexing sources with different QoS requirements together.

We have also compared the performance of three different access schemes. The results indicate the trade-off between high and low priority traffic. The insensitivity of low priority traffic to the value of $K$ has given us some sense of the buffer allocation in a multiplexer with fixed capacity. For example, we would first determine the threshold $K_{1}$ needed to support the low priority traffic. Afterwards, we only need to find the additional buffer space $\left(K-K_{1}\right)$ to support the high priority traffic. This reduces the complexity of the problem.

Finally, we investigated the problem of resource allocation to support the QoS requirements of the given traffic. We formulate this as an optimization problem and propose an iterative algorithm to solve it. The algorithm is simple and easy to implement. Numerical results to demonstrate the effectiveness of the proposed algorithm have also been given.

\section{Appendix A}

Scheme II: refer to Table 1.

(1) $B_{x} \leq C$ :

$$
a_{k h}^{x}= \begin{cases}1 & h=\max \left(0, k-\left\lfloor\left(C-B_{x}\right) T\right\rfloor\right) \\ 0 & \text { otherwise }\end{cases}
$$

(2) $B_{x}>C$ and $B_{x}^{(1)}<C: \quad a_{k h}^{x}$ is the same as the one in Scheme I.

(3) $B_{x}>C$ and $B_{x}^{(1)} \geq C: \quad a_{k h}^{x}$ is the same as the one in Scheme I. 
Scheme III:

(1) $B_{x} \leq C: \quad a_{k h}^{x}$ is the same as the one in Scheme II.

(2) $B_{x}>C$ and $B_{x}^{(1)}<C$ :

$$
a_{k h}^{x}= \begin{cases}1 & k \leq K_{1}, \quad h=\min \left(K_{1}, k+\left\lfloor\left(B_{x}-C\right) T\right\rfloor\right) \\ 1 & k>K_{1}, \quad h=k \\ 0 & \text { otherwise }\end{cases}
$$

(3) $B_{x}>C$ and $B_{x}^{(1)} \geq C: \quad a_{k h}^{x}$ is the same as the one in Scheme I.

\section{Appendix B}

The transition matrix of the video sources are shown below:

$$
\mathbf{P}^{1}=\left[\begin{array}{cccccccc}
0.803 & 0.171 & 0 & 0.013 & 0 & 0.013 & 0 & 0 \\
0.027 & 0.928 & 0.032 & 0.002 & 0.005 & 0.002 & 0.004 & 0 \\
0 & 0.058 & 0.907 & 0.035 & 0 & 0 & 0 & 0 \\
0 & 0.003 & 0.031 & 0.935 & 0.026 & 0.003 & 0 & 0.002 \\
0 & 0 & 0.005 & 0.031 & 0.944 & 0.017 & 0.003 & 0 \\
0 & 0 & 0 & 0.004 & 0.063 & 0.87 & 0.059 & 0.004 \\
0 & 0 & 0 & 0 & 0.006 & 0.109 & 0.885 & 0 \\
0 & 0 & 0 & 0 & 0.008 & 0 & 0.008 & 0.984
\end{array}\right]
$$

$$
\mathbf{P}^{2}=\left[\begin{array}{cccccccc}
0.805 & 0.195 & 0 & 0 & 0 & 0 & 0 & 0 \\
0.077 & 0.808 & 0.115 & 0 & 0 & 0 & 0 & 0 \\
0 & 0.12 & 0.759 & 0.119 & 0.002 & 0 & 0 & 0 \\
0 & 0.002 & 0.132 & 0.721 & 0.144 & 0.001 & 0 & 0 \\
0 & 0 & 0.002 & 0.138 & 0.746 & 0.113 & 0.001 & 0 \\
0 & 0 & 0 & 0.004 & 0.179 & 0.754 & 0.063 & 0 \\
0 & 0 & 0 & 0 & 0.004 & 0.244 & 0.706 & 0.046 \\
0 & 0 & 0 & 0 & 0 & 0 & 0.282 & 0.718
\end{array}\right]
$$




\section{References}

[1] A. Baiocchi et. al., "Stochastic fluid analysis of an ATM multiplexer loaded with heterogeneous On-Off sources: an effective computational approach”, INFOCOM 92, pp. 405-414.

[2] M. Ghanbari, “Two-layer coding of video signals for VBR networks", IEEE J. Selected Area Commun., Vol. 7, No. 5, June 1989, pp. 771-781.

[3] A. Elwalid and D. Mitra, "Fluid Models for the analysis and design of statistical multiplexing with loss priorities on multiple classes of bursty traffic.”, INFOCOM 92, pp. 415-424.

[4] H. Heeke, "A traffic-control algorithm for ATM networks", IEEE Trans. Circuits \& Systems for Video Techn., Vol. 3, No. 3, June 1993, pp. 182-189.

[5] H. Kroner, "Comparative performance study of space priority mechanisms for ATM Networks.”, INFOCOM 90, pp. 1136-1142.

[6] J. G. Kemeny and J. L. Snell, "Finite Markov Chains”, Springer-Verlag, New York, 1976.

[7] F. Kishino et. al., "Variable bit-rate coding of video signals for ATM networks", IEEE J. Selected Area Commun., Vol. 7, No. 5, June 1989, pp. 801-806.

[8] P. Pancha and M. El Zarki, "Bandwidth allocation schemes for variable-bit-rate MPEG sources in ATM networks", IEEE Trans. Circuit \& Systems for Video Techn., Vol.3, No. 3, June 1993, pp. 190-198.

[9] P. Skelly and M Schwartz, "A Histogram-based model for video traffic behavior in an ATM multiplexer", INFOCOM 92, pp. 95-104.

[10] S. Tubaro, "A two layers video coding scheme for ATM networks", Signal Processing: Image Communication, Vol. 3, June 1991, pp.129-141.

[11] CCITT, "Recommendation H.261-video codec for audiovisual services at $\mathrm{p} \times 64 \mathrm{kbit} / \mathrm{s}$," Dec. 1990. 


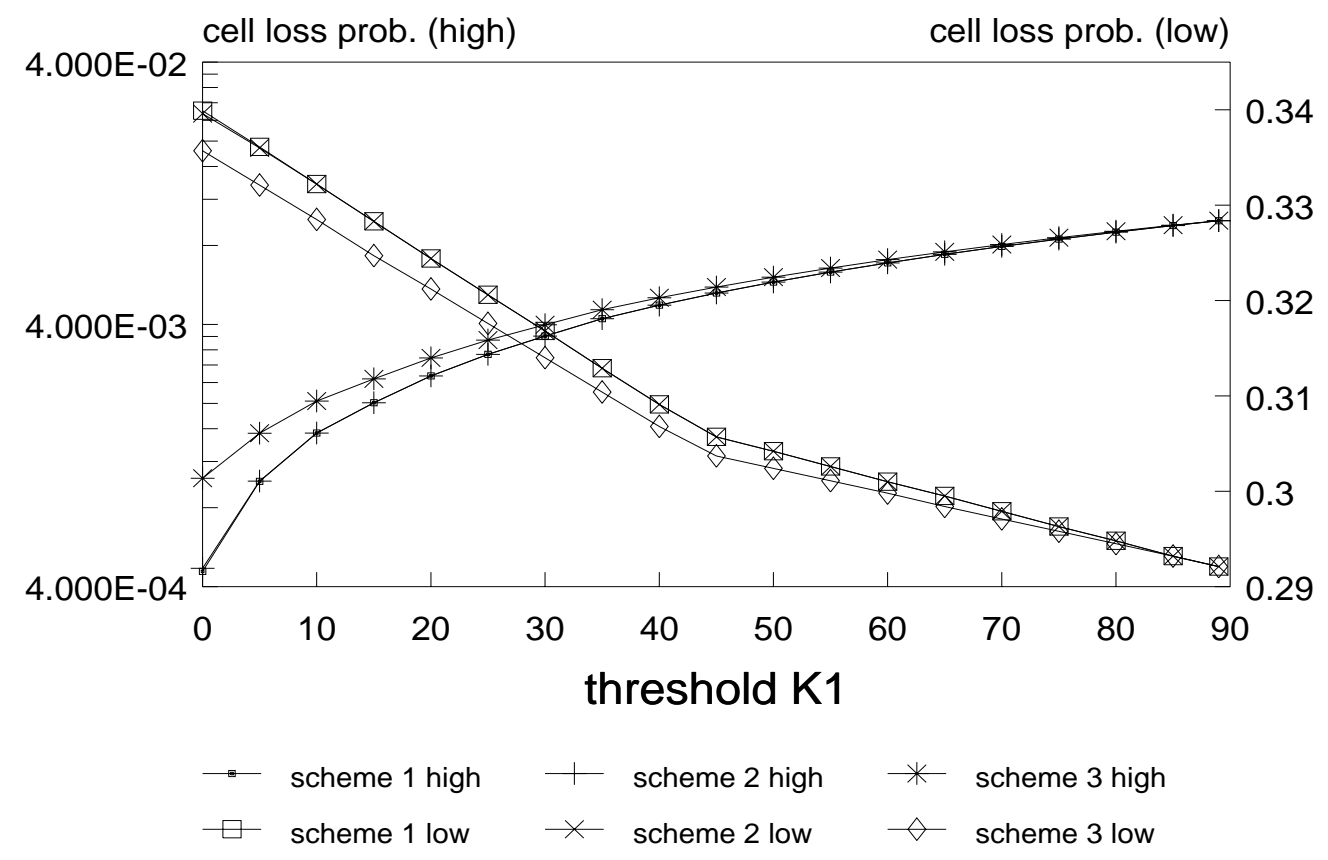

Fig. 1 Cell loss probabilities of priority streams vs. $K_{1}$ with $C=16630$ cells/s, $K=90$ cells, $\mathrm{M}=8 ; B_{x}^{(1)}=(1.0,2.0,3.0,4.0,5.0,6.0,7.0,8.0) \mathrm{Mb} / \mathrm{s}$, $B_{x}^{(2)}=(0.5,1.0,1.5,2.0,2.5,3.0,3.5,4.0)$ and $\mathbf{P}=\mathbf{P}^{1}$. 


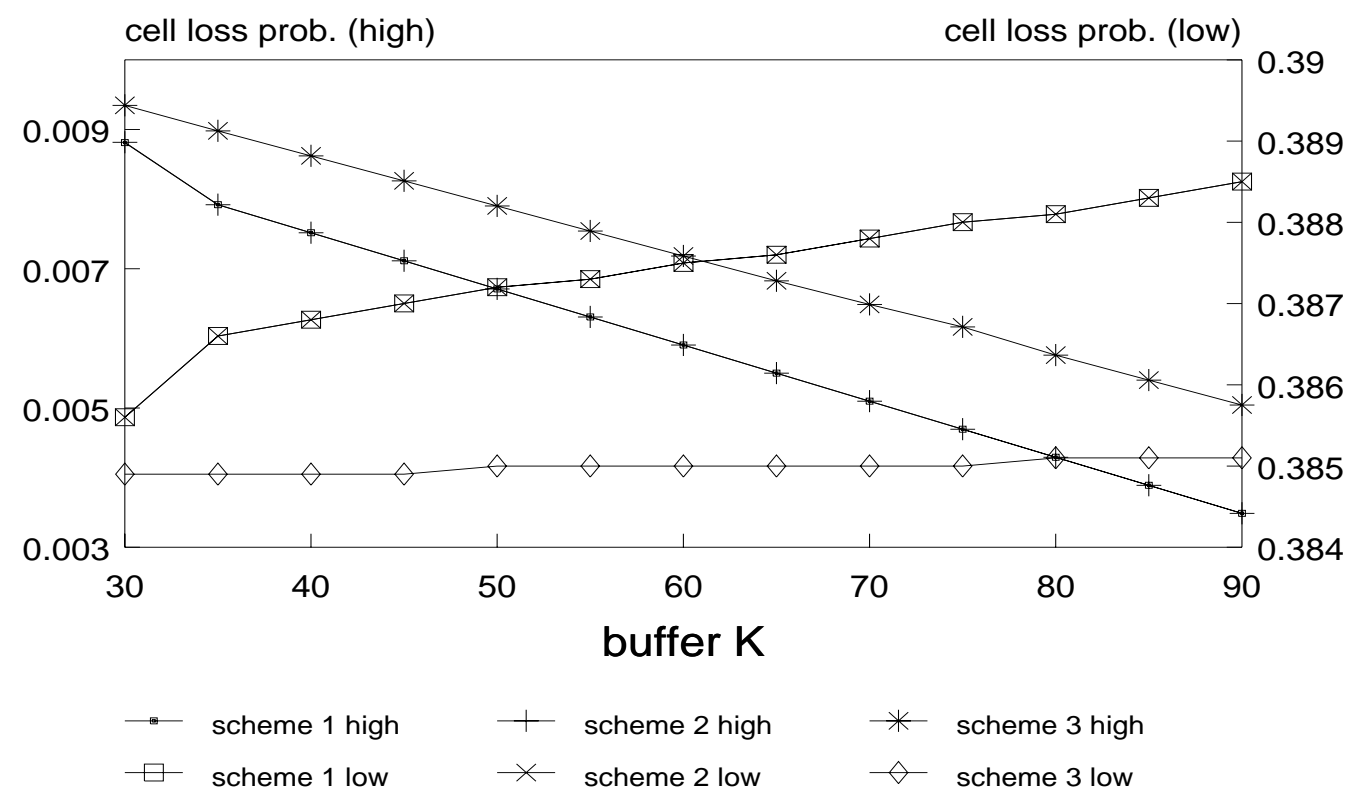

Fig. 2 Cell loss probabilities of priority streams vs. $K$ with $C=20990$ cells $/ \mathrm{s}$ $K_{1}=25$ cells, $\mathrm{M}=8 ; B_{x}^{(1)}=(3.0,4.0,5.0,6.0,7.0,8.0,9.0,10.0) \mathrm{Mb} / \mathrm{s}$, $B_{x}^{(2)}=(1.5,2.0,2.5,3.0,3.5,4.0,4.5,5.0)$ and $\mathbf{P}=\mathbf{P}^{1}$. 